\title{
铅系超导体中掺氟效应 *
}

\author{
蒋淑芬 高孝恢贾顺连 赵柏儒 \\ (中南工业大学化学系, 长沙 410083) (中国科学院物理研究所, 国家超导实验室, 北京 100080)
}

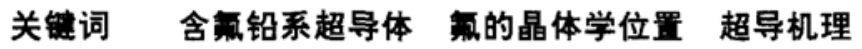

氧化物高温超导体中 $\mathrm{F}$ 代 $\mathrm{O}$ 的研究, 近来受到更多的关注. 实验结果显示, 只要制样的 方法适宜, $\mathrm{F}$ 代 $\mathrm{O}$ 都有利于超导性. 如 $\mathrm{Y}$-系及 $\mathrm{Tl}$-系, 用 $\mathrm{F}$ 代部分 $\mathrm{O}$ 使超导相更易形成, $T_{\mathrm{c}}$ 略 有提高. $\mathrm{Bi}$-系掺 $\mathrm{F}$, 有使 $T_{\mathrm{c}}$ 显著提高的效果. 属绝缘化合物的 $\mathrm{M}_{2} \mathrm{CuO}_{4}(\mathrm{M}=\mathrm{Nd}, \mathrm{Pr}, \mathrm{La})$, 当 以 $\mathrm{F}$ 代部分 $\mathrm{O}$ 时, 变为 $T_{\mathrm{c}}=30 \sim 40 \mathrm{~K}$ 的超导体. 金属性的 $\mathrm{TlSr}_{2} \mathrm{CaCu}_{2} \mathrm{O}_{7}$ 及半导体的 $\mathrm{Tl}-$ $\mathrm{Sr}_{2} \mathrm{ReCu}_{2} \mathrm{O}_{7}$ ( $\mathrm{Re}$ 为稀土) 在掺 $\mathrm{F}$ 之后, 成为 $T_{\mathrm{c}} \geqslant 77 \mathrm{~K}$ 的超导体. 近来有文献指出, 要在 $6 \mathrm{GPa}$ 高压下合成才具有超导性的 $\mathrm{Sr}_{2} \mathrm{CuO}_{3}$, 当以 $\mathrm{F}$ 代部分 $\mathrm{O}$ 时, 在常规条件下制备样品就成为 $T_{\mathrm{c}}=46 \mathrm{~K}$ 的超导体.

$\mathrm{Pb}$-系在结构上颇有特点, 自发现 ${ }^{[1]}$ 之后, 已对它进行了多方面的研究. 但是, 至今尚无在 该体系中掺 $\mathrm{F}$ 的研究报道. 对这方面进行深入的研究, 为了解超导机理是有意义的, 本文报 道了研究的初步结果.

\section{1 样品制备与测量结果}

\section{1 标准样品 $(\mathrm{A})$ 的制备}

根据名义成分 $\mathrm{Pb}_{2} \mathrm{Sr}_{2} \mathrm{Y}_{0.5} \mathrm{Ca}_{0.5} \mathrm{Cu}_{3} \mathrm{O}_{8+}$ 取 $\mathrm{Y}_{2} \mathrm{O}_{3}, \mathrm{CuO}, \mathrm{SrCO}_{3}, \mathrm{CaCO}_{3}$ 粉末研磨混合, 于 $860^{\circ} \mathrm{C}$ 预烧 $14 \mathrm{~h}$. 冷却后与 $\mathrm{PbO}$ 一起研磨均匀, 压片后分别在含 $5 \%$ 及 $2 \% \sim 0.1 \%$ 的 $\mathrm{O}_{2}$ 之氮 气流中, 于 $920^{\circ} \mathrm{C}$ 烧结 $2 \mathrm{~h}$. 然后降低温度至 $860^{\circ} \mathrm{C}$ 继续烧结 $16 \mathrm{~h}$, 取出于 $\mathrm{N}_{2}$ 中淬火.

\section{2 掺 $\mathrm{F}$ 样品 $(\mathrm{B})$ 的制备}

按名义成分 $\mathrm{Pb}_{2} \mathrm{Sr}_{2} \mathrm{Y}_{0.5} \mathrm{Ca}_{0.5} \mathrm{Cu}_{8-y} \mathrm{~F}_{y}(y=0.5 \sim 2.5 \mathrm{~mol})$ 取 $\mathrm{Y}_{2} \mathrm{O}_{3}, \mathrm{CuO}, \mathrm{CuF}_{2}, \mathrm{SrCO}_{3}$ 及 $\mathrm{CaCO}_{3}$ 混合, 于 $860^{\circ} \mathrm{C}$ 预烧 $14 \mathrm{~h}$. 冷却后加入 $\mathrm{PbO}$ 并研磨均匀, 根据掺 $\mathrm{F}$ 量的不同, 选择高温烧 结 $2 \mathrm{~h}$ 及低温退火 $16 \mathrm{~h}$ 的温度列于表 1 中. 烧结的气氛与 $(\mathrm{A})$ 样相同.

\section{3 测量结果}

(1) 含 $\mathrm{F}$ 量的测定: 由于氟化物中的 $\mathrm{F}$ 在高温下是易挥发的成分, 采用离子选择电极法; 测得样品中 $\mathrm{F}$ 的实际含量列于表 1 第 2 行.

(2)扫描电子显微镜观察: 样品的形貌在日立 X-650 SEM 上进行观察. (A) 类样品呈细条 状密堆, 随掺 $\mathrm{F}$ 量增加, 晶粒逐步增大. 当 $\mathrm{F}$ 的名义成分 $\geqslant 1.0 \mathrm{~mol}$ 时, 晶体呈片状. 图 1 中 
表 1 掺 $\mathrm{F}$ 样品 (B)的烧结与退火温度

\begin{tabular}{|c|c|c|c|c|c|}
\hline 掺 $\mathrm{F}$ 的 $\mathrm{mol}$ 数 & 0.50 & 1.00 & 1.50 & 2.00 & 2.50 \\
\hline 样品中实际含 F 量 & - & 0.63 & 0.98 & 1.12 & 1.44 \\
\hline 烧结 $2 \mathrm{~h}$ 的温度 $\mathrm{C}$ & 900 & 890 & 880 & 870 & 870 \\
\hline 退火 $16 \mathrm{~h}$ 的温度 $\mathrm{C}$ & 860 & 850 & 845 & 845 & 840 \\
\hline
\end{tabular}

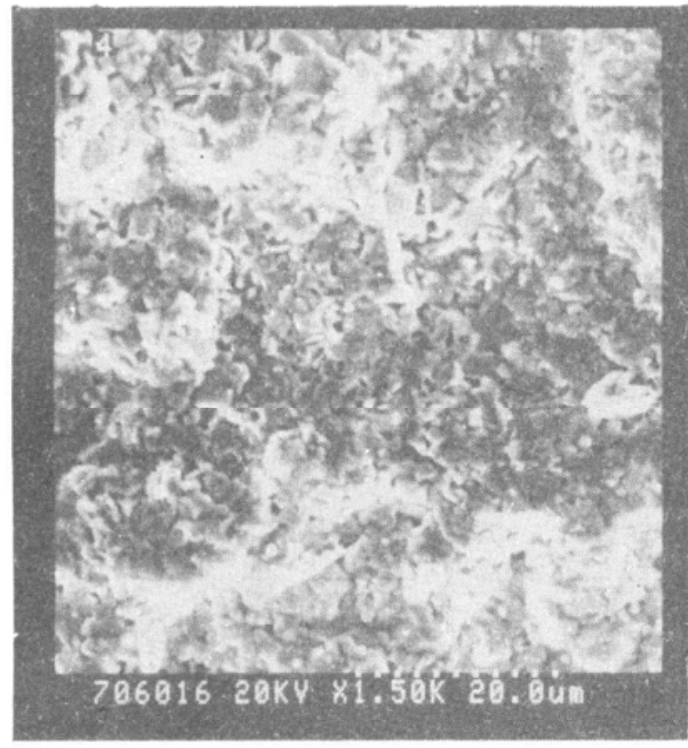

(A)

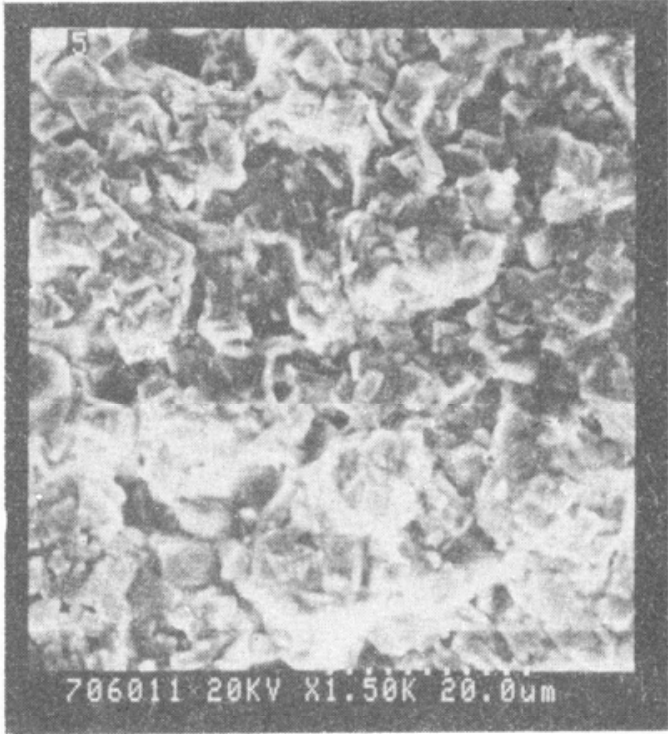

(B)

图 1 (A), (B)样品的形貌

(B) 为 $\mathrm{F}$ 的名义成分等于 $2.0 \mathrm{~mol}$ 样品的照片, 对比 图 1 中 $(\mathrm{A})$ 和 (B) 可见, $\mathrm{F}$ 的掺入有利于晶体的发育和 生长.

(3) X 射线衍射: 样品的 X 射线分析在 SIEMENS $D 500$ 衍射仪上进行. 当掺 $F \geqslant 2.0 \mathrm{~mol}$ 时, 除了可以 检索到痕量的 $\mathrm{SrF}_{2}$ 之外, 无其他杂相存在. 图 2(A) 及(B)分别为标准及掺 $\mathrm{F}=2.0 \mathrm{~mol}$ 样品的衍射花样, 它们极为相似. (A) 样的晶格参数 $a=0.5387 \mathrm{~nm}, b$ $=0.5444 \mathrm{~nm}, c=1.5688 \mathrm{~nm}$. (B)样的 $a=0.5371$ $\mathrm{nm}, b=0.5406 \mathrm{~nm}, c=1.5673 \mathrm{~nm} . V_{\mathrm{A}} / V_{\mathrm{B}}=460$. $08 / 455.08=1.011$, 可见掺 $\mathrm{F}$ 样品元胞体积有所压 缩.

(4)样品的超导电性: R-T 曲线由标准的四引线 法, $\chi-T$ 曲线由感应法测量. 结果显示, 其 $T_{\mathrm{c}}$ 与烧结 气氛及掺 $\mathrm{F}$ 量有关. 在空气中烧结的所有样品为绝 缘体. 于 $3 \% \leqslant \mathrm{O}_{2} \leqslant 5 \%$ 的氮气流中烧结, 其所有样 品的 $\dot{R}-T$ 曲线呈半导体行为, 但标准样品有零电阻

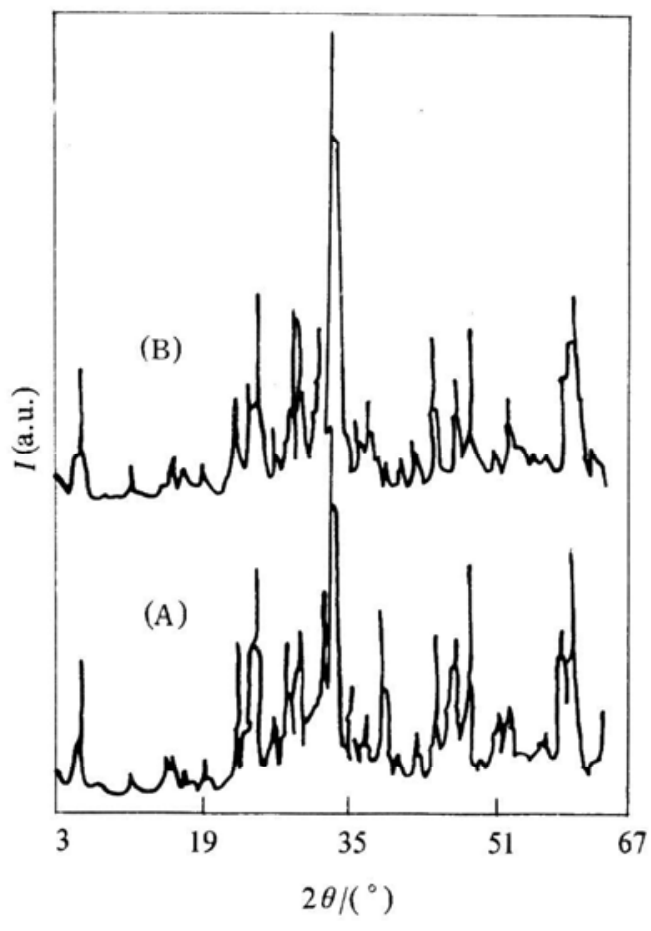

图 2 样品(A)及掺 $\mathrm{F}=2.0 \mathrm{~mol}$ 的 (B)样品 X 射线衍射图 
温度 $T_{\mathrm{CO}}=41.0 \mathrm{~K}$, 掺 $\mathrm{F}=2.0 \mathrm{~mol}$ 样品的 $T_{\mathrm{CO}}=52.0 \mathrm{~K}$. 它们的 $R-T$ 及 $\chi-T$ 曲线分别如图 3 中 (a), (b), (c)及 (d) 所示, 体系掺 $F=2.0 \mathrm{~mol}$ 为最佳.

如果样品在 $\mathrm{O}_{2} \leqslant 2 \%$ 的氮气中烧结, (A) 类样品的 $R-T$ 曲线略呈半导体型如图 3 中 (A), 而掺 $\mathrm{F} \geqslant 0.5 \mathrm{~mol}$ 样品 (B) 的 $R-T$ 曲线呈金属性, 图 3 中曲线 (B) 是掺 $\mathrm{F}=2.0 \mathrm{~mol}$ 样品的 $R-T$ 曲线, 其 $T_{\mathrm{c}}=76.2 \mathrm{~K}$, 比标准样品 $(\mathrm{A})$ 的 $T_{\mathrm{c}}=62.0 \mathrm{~K}$ 提高约 $14 \mathrm{~K}$.

(5)红外光谱测定: 为了解 $\mathrm{F}$ 是否进入超导相, 处于何晶体学位置, 我们用 Nicolet $5 \mathrm{pc}$ 红 外吸收仪; 分别测量了标准样品 (A) 及掺 $\mathrm{F}=2.0 \mathrm{~mol}$ 样品 (B), 并以 $\mathrm{CuF}_{2}, \mathrm{SrF}_{2}, \mathrm{CaF}_{2}, \mathrm{YF}_{3}$ 及 $\mathrm{PbF}_{2}$ 等化合物为参照. 两类样品的红外吸收曲线如图 4 中 $(\mathrm{A})$ 及 $(\mathrm{B})$ 所示. 曲线 (B) 显示有 两个 $\mathrm{Cu}-\mathrm{F}$ 键振动吸收峰, 它们分别位于 490.23 和 $470.13 \mathrm{~nm}$. 还有 $\mathrm{Sr}-\mathrm{F}$ 键振动吸收峰, 位于 $460.90 \mathrm{~nm}$.

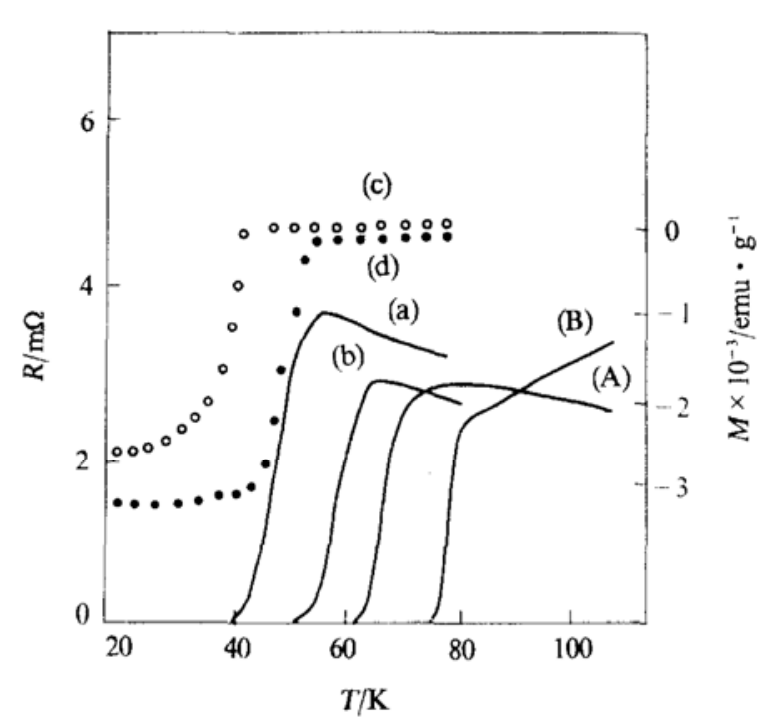

图 3 (A)及(B)样品的 $\chi-T, R-T$ 曲线

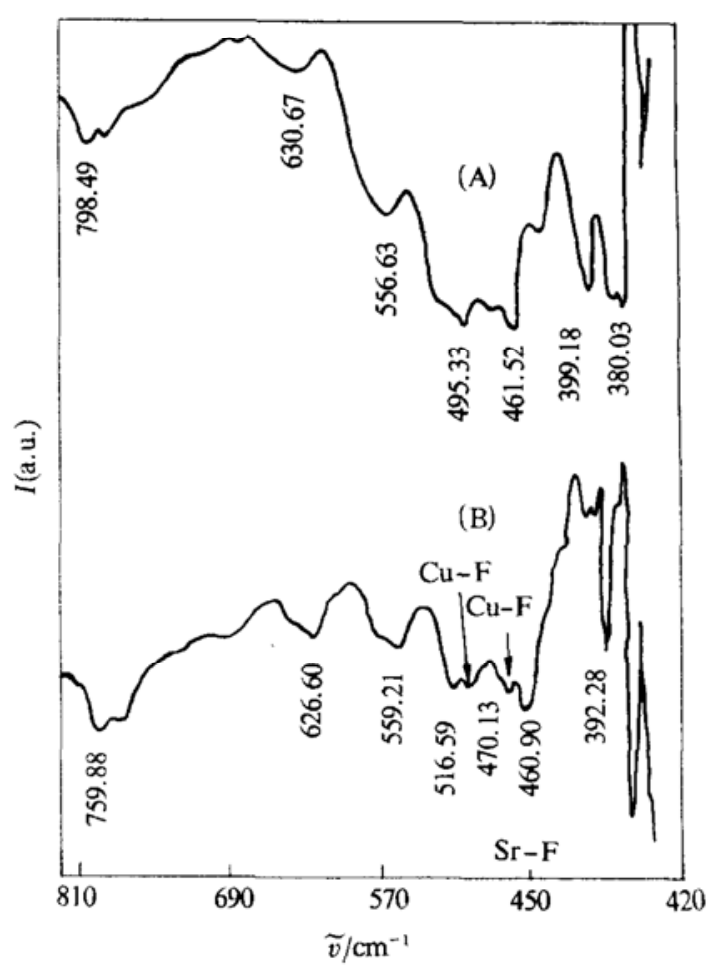

图 4 (A)和 (B) 样品的红外吸收光谱图

\section{2 讨论与结论}

\subsection{F 是否进入了超导相}

$\mathrm{F}$ 的测定结果显示, 烧结后的 (B) 样品中还含 $1.12 \mathrm{~mol}$ 的 $\mathrm{F}$. 但是 $\mathrm{X}$ 射线分析未找到任 何显著的氟化物杂相存在, 同时 (B) 样的晶格参数明显变小, 这两方面的结果即意味着 $\mathrm{F}$ 进入 了超导相. 此外, 掺 $\mathrm{F}$ 样品使 $T_{\mathrm{c}}$ 显著提高是 $\mathrm{F}$ 进入超导相的另一迹象. 清晰的 $\mathrm{Cu}-\mathrm{F}$ 键及 $\mathrm{Sr}-\mathrm{F}$ 键红外吸收峰, 是 $\mathrm{F}$ 进入超导相的进一步证据.

\section{$2.2 \mathrm{~F}$ 的晶体学位置}

红外光谱显示, 掺 $\mathrm{F}$ 样品中既存在 $\mathrm{Sr}-\mathrm{F}$ 键振动峰, 又存在波数不同的两个 $\mathrm{Cu}-\mathrm{F}$ 键振动 峰, 这种现象说明 $\mathrm{F}$ 在元胞中至少有两种占位. 根据计算固体力常数 $K$ 的公式 ${ }^{[2]}$ : 


$$
K^{-1 / 2}=a_{\mathrm{ij}}\left(D_{e}-b_{\mathrm{ij}}\right),
$$

其中 $a_{\mathrm{ij}}$ 及 $b_{\mathrm{ij}}$ 是随键合两元素在周期表中的行数有关的常数, $D_{\mathrm{e}}$ 是 $\mathrm{ij}$ 两元素的平衡核间距. 由该公式估算结果能够指出, 长波峰为占据顶 $\mathrm{O}$ 位置的 $\mathrm{F}$ 并与 $\mathrm{Cu}$ 成键的振动所产生. 这种 晶位上的 $\mathrm{F}$ 正好位于 $\mathrm{SrO}_{2}$ 面上,并易与 $\mathrm{Sr}$ 成键.

上述公式估算显示, 另一 $\mathrm{Cu}-\mathrm{F}$ 键振动峰, 为 $\mathrm{F}$ 取代 $\mathrm{CuO}_{2}$ 面上的 $\mathrm{O}$ 形成 $\mathrm{Cu}-\mathrm{F}$ 键所产生. 因为 $\mathrm{F}$ 离子半径 $r_{\mathrm{F}^{1-}}=0.136 \mathrm{~nm}$, 比 $\mathrm{O}$ 离子半径 $r_{\mathrm{O}^{2-}}=0.14 \mathrm{~nm}$ 明显偏少. 从得到的晶格参 数来看, 掺 $\mathrm{F}$ 后 $b$ 轴收缩明显, 所以取代 $\mathrm{CuO}_{2}$ 面上 $\mathrm{O}$ 位的 $\mathrm{F}$ 应处于 $b$ 轴方向.

\section{3 改善超导性的机理}

$\mathrm{Pb}$-系是空穴载流子超导体 ${ }^{[3]}$, 其中 $\mathrm{CuO}_{2}$ 面是超导电流负载者, $\mathrm{PbO}_{2}$ 为电荷贮存层. 这 两种面由一个共用 $\mathrm{O}$ 原子连接成为四方双雉, 即 $\mathrm{CuO}_{4}-\mathrm{O}-\mathrm{PbO}_{4}$ 结构单位. 上面指出 $\mathrm{F}$ 占据 $\mathrm{CuO}_{5}$ 中的顶角氧位就是这种共顶氧, 它具有控制 $\mathrm{CuO}_{2}$ 面上载流子浓度的关键作用. 当以 $\mathrm{F}$ 占据该晶体学位置时, 由于 $\mathrm{F}$ 吸引电子能力较大而使 $\mathrm{CuO}_{2}$ 面上载流子浓度增加, 于是有利于 $T_{\mathrm{c}}$ 的提高. 这种机理,在掺 $\mathrm{F}$ 铋系中得到量子化学簇模型 $X_{\alpha}$ 方法计算认可 ${ }^{[4]}$.

文献 [5]分析指出, 所有空穴型铜基氧化物超导体, $\mathrm{CuO}_{5}$ 雉体中顶角氧对 $\mathrm{CuO}_{2}$ 面电子态 具有基本的重要性. 其顶角氧 Medelung 势 $V_{\mathrm{O}(\mathrm{A})}$ 与平面氧的 Madelung 势 $V_{\mathrm{O}(\mathrm{P})}$ 之差 $\Delta V_{\mathrm{A}}$ 越 大, 该超导体的 $T_{\mathrm{c}}$ 愈高. $\Delta V_{\mathrm{A}}$ 可以由 $\mathrm{Cu} 3 d$ 的晶体场分裂能估算. $\mathrm{F}^{1-}$ 与 $\mathrm{O}^{2-}$ 为等电子体 系, 但是前者半径更小, 有使 $\mathrm{Cu} 3 d$ 能级分裂更大的效果, 所以由 $\mathrm{F}$ 代 $\mathrm{O}$ 后, 使 $T_{\mathrm{c}}$ 显著提高.

由 (A)及 (B) 两类样品的晶格参数可见, 掺 $\mathrm{F}$ 使元胞增加了内压, 这也是 $(\mathrm{B})$ 样品 $T_{\mathrm{c}}$ 提高 的原因之一.

\section{4 结论}

在 $\mathrm{Pb}$-系超导体中掺 $\mathrm{F}, \mathrm{F}$ 进入了超导相, 分别占据 $\mathrm{CuO}_{5}$ 雉体中顶 $\mathrm{O}$ 及平面 $\mathrm{O}$ 的晶体学 位置, 有利于超导相形成和使 $T_{\mathrm{c}}$ 明显提高的效果.

\section{参考文献}

1 Cava R J, Batlogg B, Krajewski J J et al. Superconductivity near 70K in a new family of layered copper oxide. Nature, 1988 , 336: 211

2 Pauling L. The Nature of the Chemical bond. Cornell: Cornell Univ Press, 1960

3 Sunagaw $\mathrm{H}$, Kaiwa M, Koike $\mathrm{Y}$ et al. Hall effect and substitution effect in $\mathrm{Pb}_{2} \mathrm{Sr}_{2} \mathrm{Y}_{1-x} \mathrm{Ca}_{x} \mathrm{Cu}_{3} \mathrm{O}_{8+8}$. $\mathrm{Physica} \mathrm{C}, 1991$, 185-189:1271

4 刘洪菻, 陈念贻, 尹周澜等. 掺 $\mathrm{F}$ 提高 $\mathrm{Bi}$ 系超导体 $T_{\mathrm{c}}$ 的量子化学研究. 化学物理学报, 1992, 5:98

5 Ohta Y, Tohyama T, Maekawa S. Apex oxygen and critical temperature in copper oxide superconductors: Universal Correlation with the stability of local singets. Phys Rev B, 1991, 43: 2968 